\title{
The Study on Technology and Application of laaS in Campus Network
}

\author{
Huixiao Wang \\ School of Leisure Management, Xi'an eurasia University , Shaanxi , Xi'an 710065 ,China \\ wxh2324@126.com
}

Keywords: laaS; VDI; Virtualization; Cloud computing

\begin{abstract}
Currently, the network in many universities is developing rapidly. But the utilization is low and the inadequate of servers in construction. So, this paper introduces a server virtualization technology based on VDI technology taking Xi'an Eurasia University as the instance. This technology will be implemented in the school network and get a good effect.
\end{abstract}

\section{Introduction}

In the recent years, as cloud computing technology is widely developed and applied, IT equipment manufacturers, Internet companies, software companies and even telecom operators have given high attention[1]. According to the authoritative definition of NIST and the most widely recognized division in the industry, cloud computing has SPI, which have the three major service modes of SaaS, PaaS and IaaS. IaaS (Infrastructure as a Service), defined the infrastructure as a service and provide consumers with services of all facilities, including the processing, storage, network and other basic computing resources. Users can deploy and run arbitrary software, including operating system and application program. Although consumers do not need to manage or control any cloud computing infrastructure, they can control the choice of operating systems, or storage space and deployment applications[2].

The IaaS services will be able to do resources monitoring, dynamic dispatching and optimization, virtual machine online migration, physical machine load balancing, high availability, virtual machine online backup and recovery, network bandwidth distribution, storage backup, etc. Among them, dynamic scheduling and optimization means that when an application has insufficient resources, it can dynamically add resources to it. When an applied resource does not work, it can be recycled into the memory pool. Online migration means that when a physical machine needs downtime maintenance, the virtual machine can move to another physical machine by internet to ensure that services are not interrupted; The physical machine load equilibrium is that when a physical machine is high load, it can automatically transfer the virtual machine to a lighter physical machine on the condition of not being able to stop it; High availability means that when a physical machine fails, the virtual machine running on it can be started quickly on another physical machine to maximize the continuity of the service[3].

So, using cloud computing and virtualization technology, various resources can be constructed in a virtual manner to complete the resource sharing of application layer. Therefore, the university can save the hardware procurement and improve the utilization efficiency of hardware resources.

\section{The Problem of Campus Network in Colleges and Universities}

With the rapid development of education in recent years, the original network resources of the school are obviously inadequate. For example:

The teaching management system of students' elective course has only three servers. After the semester starts, more than 30,000 students will be enrolled in the educational administration system. All of the students in the middle of the semester have to log on to the administration system in a few days to grade a teacher. At the end of the term, the teacher needs to record the student's performance in a few days. The new enrollment system, one server, each year the incoming new students' information needs to be recorded; It is inevitable that the equipment utilization rate will be low and the resource will be wasted, if the system and the new enrollment system are simply increase to cope with the large concurrent amount of access. 
At the same time, many hardware resources are not available at school, so resources are left idle. For example: recruitment network (one server idle time: October to march); Tsinghua teaching system (two servers are idle); Psychological evaluation system (one server, $1 \sim 2$ times per semester, most of the time is idle)(Table 1).

Table 1 Example of idle resources

\begin{tabular}{|c|c|c|}
\hline Resources & Number & State \\
\hline Enrolment network & 1 servers & $\begin{array}{c}\text { It runs from April to September every year, } \\
\text { and the rest of the time is idle. }\end{array}$ \\
\hline Tsinghua teaching system & 2 servers & Inactive \\
\hline Psychological evaluation system & 1 servers & $\begin{array}{c}\text { Use 1 2 times per semester, most of the } \\
\text { time is idle }\end{array}$ \\
\hline
\end{tabular}

According to the actual situation, it is necessary to make full use of the existing resources of the network center .Using cloud computing technology to build a shared server cluster. It provides IaaS services and gradually uses the servers of all application systems in the school. It gradually realizes resource sharing solves the problem of large number of visits and improves the utilization of hardware equipment[4].

\section{Virtualization Technology}

The concept of virtualization was first proposed by IBM in the 1970s. The realization of software virtualization technology mainly includes three ways: full virtualization, partial virtualization and operating system layer virtualization. Their difference is that the Virtual Machine Monitor (VMM) has a different location, function, and implementation.

Full Virtualization. Fully virtualized technology is used to manage each virtual machine by creating an abstract layer of virtual machine monitoring layers between the virtual machine and the host machine (Host) hardware. Through the VMM layer, full virtualization technology simulates the complete virtual hardware platform of multiple BIOS levels, and multiple heterogeneous operating system virtual machines can be run on single hardware platform. The virtual machine is completely transparent to the client operating system. The drawback of full virtualization is that the VMM mid-tier code transformation can bring some cost to the host. The technology is mainly represented by EMC's VMware and Microsoft's virtual server[5].

Partial Virtualization. Partial virtualization is also called quasi virtualization. In order to reduce the burden of host computers, partial virtualization technology customize the kernel of the client operation system by using the virtualization application interface (API) to replace some X86 privileged instructions that cannot be virtual, so that it can work with the VMM layer to enhance the performance of the virtual machine[6]. The disadvantage of partial virtualization is that the virtual machine is not completely transparent to the client operating system.

Operating System Virtualization. The concept of operating system virtualization is to copy multiple client operating systems to run mirrors in a public operating system[7]. The operating system virtualization technology has no independent VMM virtualization processing layer, and provides a thinner virtual architecture system. The virtualization method has high efficiency, but it can not implement the dissimilar operating system client virtual machine deployment on the single host computer. SWsoft, Virutozzo and Sun's Solaris Container use virtualization technology operating system.

CPU Assisted Virtualization. Virtualization technologies support multiple client operating systems in a host machine and require the VMM software layer to adjust the client's application for host CPU, memory, and I/O resources. The CPU of a traditional X86 architecture is not designed to support multiple operating systems in parallel. The privileged instructions required for operation of the client operating system depend on the VMM software layer simulation implementation, which will result in 
loss of performance. The importance of the development of virtualization technology enables both Intel and AMD to redesign the X86 architecture providing hardware assisted virtualization technology to improve the efficiency of the virtual machine.

The development and importance of virtualization technology has enabled both Intel and AMD to redesign the X86 architecture. They provide hardware assisted virtualization technology to improve the efficiency of virtual machines.

\section{Selection of Virtual Software Platform}

Consideration of Virtualization Technology. The use of virtualization technology in the data center needs to take full account of the following five factors:

The application system needs for CPU, memory and I/O devices: to migrate an application

system to the virtual machine, we need to evaluate the application's demand for computer resources. The continuous application of video resources such as video services is not suitable for migration to the virtual machine.

The number of physical servers to be integrated: the relationship between the number of

integrated services and the cost to price of the host hardware needs to be balanced;

The type of operating system of virtual machine running: whether it needs the virtual machine of various operating systems to run at the same time;

The virtualization technology; (5) virtual machine implementation of the software cost.

Comparison of Virtualization Technology. TVirtualization technology to create cloud computing center is crucial, virtualization technology is a key technology of cloud computing, cloud computing as a host to run many applications at the same time, the processing power and storage space can be different according to the demand of application sharing. Virtualization technology can expand the capacity of the hardware and simplify the reconfiguration process of the software. Virtualization technology enables multiple virtual servers to run on a single computer, that is, many operation systems are running, and there are multiple programs running in every operation system. Every operation system runs on a virtual CPU or virtual host. So that all the applications can run in the independent space and do not affect each other, which greatly improves the efficiency of the computer[8].

The three ways to implement virtualization technology are isolation virtualization and compatibility: full virtualization, partial virtualization, operation system level virtualization, and virtualization of virtual machines, partial virtualization, operation system layer virtualization. Isolation and operation benefit are two main factors that must be considered when virtualization technology is implemented. All kinds of technological ways are seeking balance points in these contradictory factors.

Complete virtualization has good isolation, compatibility and flexibility, and the disadvantage is that the VMM software layer code conversion will bring a certain amount of system overhead. The emergence of CPU assisted virtualization hardware technology, complete virtualization from software layer simulation function has gradually turned to hardware, and the efficiency of virtualization is increasing. With the continuous improvement of CPU assisted virtualization technology, complete virtualization technology has a broad prospects for development.

Selection of Virtualization Software. SUNDE Diana is the Hui Yuan Electronic Technology Limited company's products, is running the vPoint desktop virtualization software on the host platform, through the SUNDE-VDI protocol, do not rely on third party software VDI and GPU, users in the Diana terminal, perfect only $5 \mathrm{~W}$ power on the virtual desktop experience, support all x86 operating system, PC machine the perfect run almost all of the software, to provide users with "HD experience". Diana is the only cloud computing terminal integrated with VDI software and hardware technology, and the technology level is in the forefront of the world[9].

The system environment built on SUNDE cloud computing terminal as client hardware platform is not only powerful and convenient to use, but also has distinct characteristics and significant advantages in system security, reliability, manageability and overall cost of ownership. Using SUNDE cloud computing terminal office automation solutions, it can support the company's data application, 
decision-making, technical information, system security and other specific needs. It also makes a breakthrough in management technology[10].

\section{Innovation Point}

At home and abroad as the cloud desktop virtualization terminal is mainly dependent on the operation of the remote desktop software in the host operating system (RDP, ICA, PCoverPC, VNC), multi user terminal technology is traditional, the user is very dependent for the host operating system version and built-in remote desktop software, and users can not lead to as the use of local physical host as smoothly, make full use of the function of the host instance. Most of the thin clients and cloud terminals in the market belong to this shared mode of remote virtual terminal products. The virtual host technology through VDI interface technology and protocol access is faster and more reliable than terminal service mode. Each virtual host is exclusive to a virtual terminal at the same time, and has better user experience. It is the mainstream technology of IaaS application in the future.

The remote virtual desktop terminal is connected to the virtual host through the VDI interface technology and protocol. The access mode is faster and more reliable than the terminal service mode, and each virtual host is exclusive to one virtual terminal at the same time. This kind of cloud computing terminal based on VDI technology is the mainstream of the future cloud computing base IaaS. Its biggest feature is the ability to use virtual hosts of all kinds of operating systems remotely, very fluently, conveniently and flexibly.

\section{Implementation of IaaS in Campus Data Center}

Make full use of existing network resources center, the use of cloud computing technology construction the shared shared server group, providing IaaS services, and gradually the application system the server, realize the sharing of resources, solve the problem of large amount of concurrent access, improve the utilization rate of hardware equipment.

Finally, make full use of the network center room environment and the operation and maintenance of power, the use of cloud computing technology construction the shared shared server group, providing IaaS services, gradually will all the departments and units of the server or eliminated, so as to realize the sharing of resources, improve equipment utilization, solve the problem of large concurrency.

Typical applications are as follows: by 10 sets of 2 servers, 1 sets of storage equipment, 1 sets of equipment, 1 sets of load balancing application layer firewall, 1 FC switches and 1 IP switches, 1 sets and 1 sets of virtualization software to set up IaaS service model management software based on the shared server cluster to access the campus Wan Zhaosu rate network to provide IaaS services to the school. It supports the operation of multiple business systems in the school. These business systems include mail management system, office automation system, student status management system, educational administration management system, DNS domain name service system, teaching resource library, video on demand, etc.

Among them, the virtual software is used to integrate the server group into a unified hardware platform. On the basis of this hardware platform, a series of virtual hosts are virtual, which support the website publishing system and the application system of the original business system. These virtual hosts have independent operating systems and applications. These systems, which support different software architectures, are highly integrated on the same hardware platform. All applications can automatically migrate between different nodes according to the load of the system. There will be no application interruption. The back end uses a double fiber switch to share a storage network, so any switch or server failure will not affect the operation of the system.

\section{Conclusion}

Through the test results, the IaaS service virtualization technology can effectively realize resource sharing in network center, reduce the increase of server number and simplify server management, and 
obviously improve server utilization, network, flexibility and reliability. In terms of the application effect, take students' elective courses as an example. During peak hours of high concurrency, through the load balancing of virtual machine, it will be able to support thousands of people to select courses online at the same time, and change the face of slow response and low service quality during peak hours.

In addition, the virtual technology can also be used in teaching and software testing environments. School administrators can use virtual machine software for operating systems, software applications, and some more complex network architectures in a very short period of time through less resource to achieve some virtual teaching and experimental test environment. It can save time, save resources and improve efficiency.

\section{Acknowledgements}

This work was partly supported by Shaanxi Province Social Science Fund Project(NO.2016R022)of China. And by The scientific research fund project of Shaanxi Provincial Department of Education.

\section{References}

[1] Mistry S, Bouguettaya A, Dong H, et al. Metaheuristic Optimization for Long-term IaaS Service Composition[J]. IEEE Transactions on Services Computing,99( 2016) 1-7.

[2] Yamato Y. Optimum Application Deployment Technology for Heterogeneous IaaS Cloud[J]. Journal of Information Processing, 25(2017) 56-58.

[3] Suthar K, Patel J. EncryScation: An Secure Approach for Data Security Using Encryption and Obfuscation Techniques for IaaS and DaaS Services in Cloud Environment[J]. Astrophysical Journal, 663(2017)799-807.

[4] Mitropoulou P, Filiopoulou E, Michalakelis C, et al. Pricing cloud IaaS services based on a hedonic price index[J]. Computing, 98(2016)1-15.

[5] Nirmala S J, Bhanu S M S. Catfish-PSO based scheduling of scientific workflows in IaaS cloud[J]. Computing, 98(2016)1-19.

[6] Teng F, Yu L, Li T, et al. Energy efficiency of VM consolidation in IaaS clouds[J]. Journal of Supercomputing, 73(2017)782-809.

[7] Entezari-Maleki R, Sousa L, Movaghar A. Performance and power modeling and evaluation of virtualized servers in IaaS clouds[J]. Information Sciences, 2017, 394-395:106-122.

[8] Gupta M K, Amgoth T. Resource-aware virtual machine placement algorithm for IaaS cloud[J]. Journal of Supercomputing, 2017(6):1-19.

[9] Renesse R V, Renesse R V, Renesse R V, et al. Supercloud: A Library Cloud for Exploiting Cloud Diversity[J]. Acm Transactions on Computer Systems, 2017, 35(2):6.

[10] Suthar K, Patel J. EncryScation: An Secure Approach for Data Security Using Encryption and Obfuscation Techniques for IaaS and DaaS Services in Cloud Environment[J]. Astrophysical Journal, 2017, 663(2):799-807. 